\title{
El IAPH celebra el II Encuentro Patrimonio de Proximidad, centrado en los cuidados del patrimonio y su comunidad
}

Los encuentros de patrimonio de proximidad, organizados por el Instituto Andaluz del Patrimonio Histórico (IAPH), constituyen una línea abierta de trabajo que pretende visibilizar las iniciativas y los agentes implicados en la activación del patrimonio cultural desde lo local, especialmente en Andalucía, para reflexionar, crear redes y compartir conocimientos y experiencias. El proyecto se inició en 2018; tuvo un segundo hito en 2019 en la Casa del Pumarejo de Sevilla; y se ha concretado en 2021 con la celebración de un segundo encuentro -virtual, por las circunstancias de la pandemia- en torno a los cuidados del patrimonio y su comunidad. En total se expusieron más de cuarenta experiencias sobre la activación del patrimonio cultural, en cuya valoración y selección intervino la denominada Comunidad de agentes redactívate.

Isabel Luque Ceballos | Dirección de Investigación y Transferencia, Instituto Andaluz del Patrimonio Histórico

URL de la contribución <http://www.iaph.es/revistaph/index.php/revistaph/article/view/5006>

Tras el I Encuentro Patrimonio de Proximidad en 2018, tuvo lugar al año siguiente una reunión en la Casa del Pumarejo (Sevilla), en la que se analizaron las aportaciones de la primera convocatoria ${ }^{1}$. Los agentes diversos implicados en la activación del patrimonio se plantearon cuatro retos: cómo trabajar la investigación aplicada al territorio y la salvaguarda del patrimonio; cómo crear un mapa de agentes equilibrado; cómo alcanzar el retorno social y la relevancia para la población local; y, finalmente, cómo conseguir una gestión relacional, compartida. Y como continuación, el II Encuentro Patrimonio de Proximidad, recientemente celebrado, ha pretendido difundir las conclusiones acerca de los retos del Pumarejo, desde una visión compartida de "hacer patrimonio" y con la intención de crear conciencia sobre el cambio de modelo en su gestión.

A este planteamiento con vocación relacional se sumó el tiempo extraño de emergencia sanitaria que vivimos, en el que la activación patrimonial se ha convertido en refugio frente a la desconexión personal, a la vez que en nexo de hiperconexión a través de las redes. El patrimonio es una forma de reencontrarnos y reconocernos y está cumpliendo en esta etapa una función de vínculo social. Por todo ello se propuso el lema "los cuidados del patrimonio y su comunidad". El esfuerzo de los agentes por mantener y mantenerse en el día a día y de las comunidades por conectarse a través del patrimonio ha supuesto una revisión y reflexión sobre el ecosistema profesional, patrimonial, del conocimiento y su soporte social. Todo ello se focalizó en un cambio de herramienta; el encuentro se adaptó al entorno virtual y apostó por una convocatoria abierta de vídeos y proyectos en torno a cuatro grandes temas: educación formal e informal; investigación aplicada al territorio; ecosistemas de agentes en el proceso patrimonial; y creatividad destinada al patrimonio y a la comunidad.

\section{Comunidad de agentes redactívate}

Con motivo de este llamamiento para el II Encuentro Patrimonio de Proximidad, se pensó cómo contar con la comunidad de agentes que se reconocieron en el I Encuentro (2018) y en la siguiente reunión del Pumarejo (2019). Para ello se solicitó la colaboración de algunos de los ponentes de las mesas redondas, ejemplos de buenas prácticas y agentes que habían contado sus experiencias, miembros de la comunidad del conocimiento, de asociaciones o colectivos profesionales y sociales y de las instituciones de la Administración pública. En definitiva, se constituyó un nuevo grupo heterogéneo, que compartió el proceso inicial de valoración de proyectos y vídeos para el II Encuentro Patrimonio de Proximidad, 


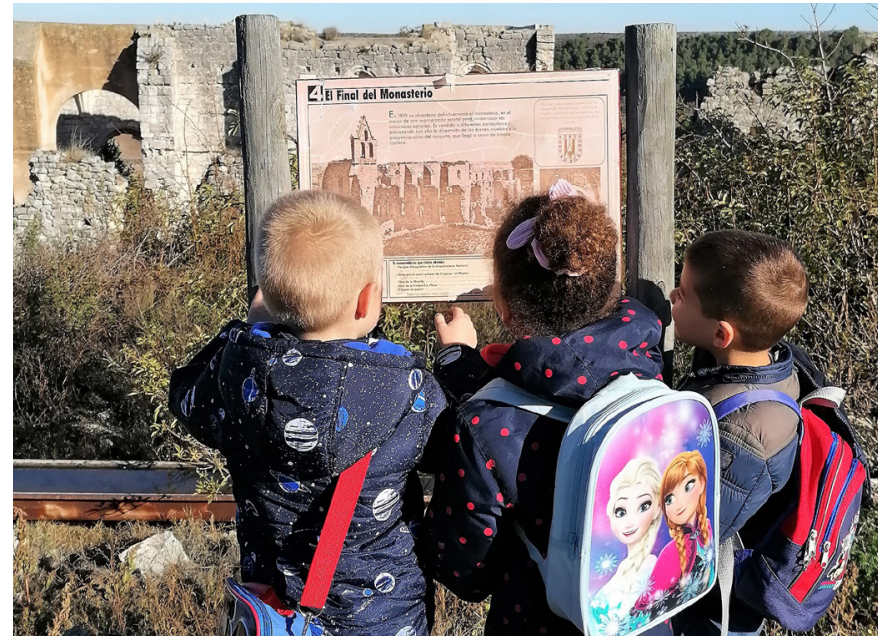

Actividades de la comunidad educativa. El calabacín errante, Valladolid | foto Marta Herrarte Sanz

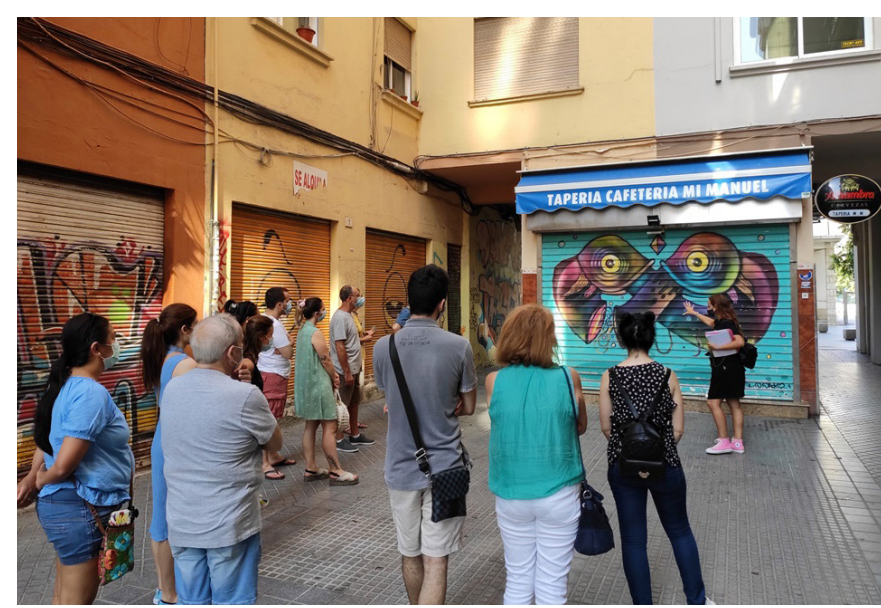

Explicando el Soho de Málaga | foto Experiencia Street Art Málaga

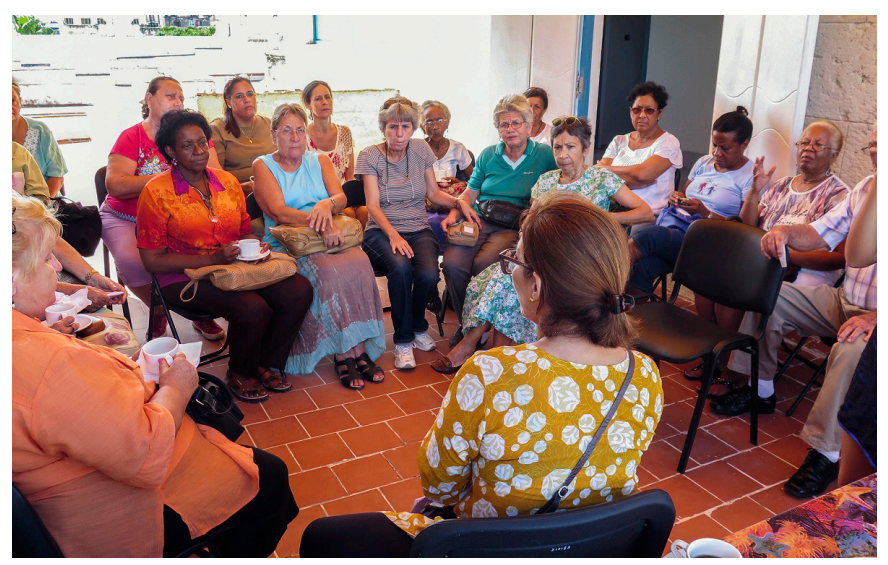

Taller de Té de intercambio, Cuba | foto Palacio del Segundo Cabo y al que agradecemos habernos acompañarnos en este camino.

En total se expusieron más de 40 experiencias -de las cuales 27 procedían de la convocatoria de vídeos y proyectos- sobre la activación del patrimonio cultural, repartidas entre las mesas redondas, el noticiario de patrimonio y el mural on line. En esta exposición ocuparon un lugar relevante las noticias sobre la actualidad de las buenas prácticas presentadas en la primera edición, para generar continuidad en el proceso y demostrar cómo las iniciativas de patrimonio pueden tener futuro. Adrián Yánez, de la Asociación Andaluza de Profesionales de la Gestión Cultural (GECA), se encargó de diseñar esta sección.

\section{Cuatro bloques temáticos}

Ángel Portolés (Proyecto Patrimoni, Universidad Jaume I) abrió el primer bloque del II Encuentro, destinado a la educación formal e informal. En su intervención nos recordó que el concepto de patrimonio cultural se estaba quedando pequeño y que, para llevarlo al límite, era necesario pensar en acompañamiento, en proyectos integrales de educación patrimonial, en proyectos comunitarios, en redes y personas. La educación desde el patrimonio supone un proceso híbrido, formal y no formal, un proceso activo y de largo recorrido, de ida y vuelta, de descubrimiento, con la participación comunitaria, no solo en la figura del visitante. Esta es la garantía de pervivencia del proyecto. En este contexto Ángel se pregunta quiénes se identifican como los expertos en estos nuevos marcos de aprendizaje y apuesta por incluir a las comunidades patrimoniales para construir este concepto de patrimonio de límites líquidos.

El patrimonio en educación constituye una forma de vida y de profesionalización, desde el marco local y municipal, una apuesta por el patrimonio inclusivo y cercano, una forma de unir relaciones, de reciprocidad; en definitiva, una herramienta para la comunidad patrimonial. Los vídeos realizados por distintas comunidades educativas que acompañaron la intervención de Portolés fueron hilvanados por Rosario Castro García (Programa Sentir y vivir el patrimonio, Consejería de Educación de la Junta de Andalucía). 
Guadalupe Jiménez Esquinas (Universidad de Santiago de Compostela) abordó el tema "Investigación y comunidad en patrimonio". Comenzó su reflexión con la idea de que somos las personas las que damos valor a los conceptos; por lo tanto, el conocimiento recorre un camino de ida y vuelta, de la sociedad para la sociedad. La comunidad debe ser partícipe del proceso de investigación, a pesar de los peligros de la participación y todo el camino que queda por hacer hasta la gobernanza. El énfasis se ha de poner en el máximo beneficio para el interés general, para el bien común. Para Jiménez Esquinas la comunidad del patrimonio la forman, con todas las cautelas y dudas que esto genera, el grupo de personas que se preocupa de que se cuide el patrimonio cultural y cree beneficios sociales. Esta reflexión fue acompañada durante el II Encuentro de una mesa redonda, coordinada por Yolanda González Campos (IAPH).

La red de personas, afectos, emociones y patrimonios correspondió a Gema Carrera Díaz (Instituto Andaluz del Patrimonio Histórico) y Rubén Alonso (Antropoloops), que hablaron al alimón de cómo el mapa se centra en el proceso social para ofrecer modelos alternativos al crecimiento socioeconómico establecido. Ambos invitaron a los agentes del territorio a participar de forma colaborativa en la Red de agentes del patrimonio cultural de Andalucía. También esta sesión estuvo acompañada de ejemplos audiovisuales sobre los cuidados de nuestro patrimonio.

"Creatividad, patrimonio y futuro" se denominó la última mesa, presentada por Ignacio Muñiz Jaén, director del Ecomuseo del río Caicena, en Almedinilla (Córdoba), y Juan López López, videocreador. El binomio contemporaneidad e historia nos conduce a la conclusión de que sin memoria, no hay futuro, pero también hace falta la creatividad para buscar caminos para abran la mente; que enriquezcan las miradas desde lo sensorial, subjetivo, simbólico y afectivo. La mesa redonda, facilitada por Valle Pérez Cano (IAPH) ayudó a tejer algunas de las experiencias presentadas.

\section{Conclusiones}

De las conclusiones extraídas durante esta intensa jornada virtual se pueden destacar que los límites del patri-

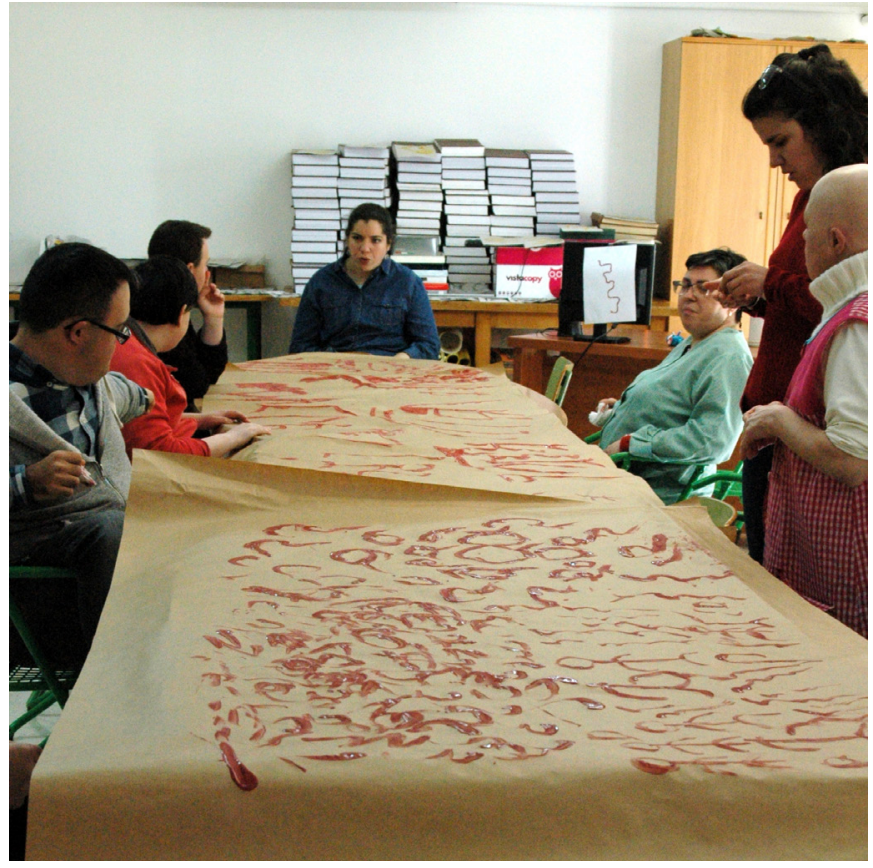

Arte capacitado. Museo de la Alcazaba, Loja (Granada) | foto Rosana Cansino

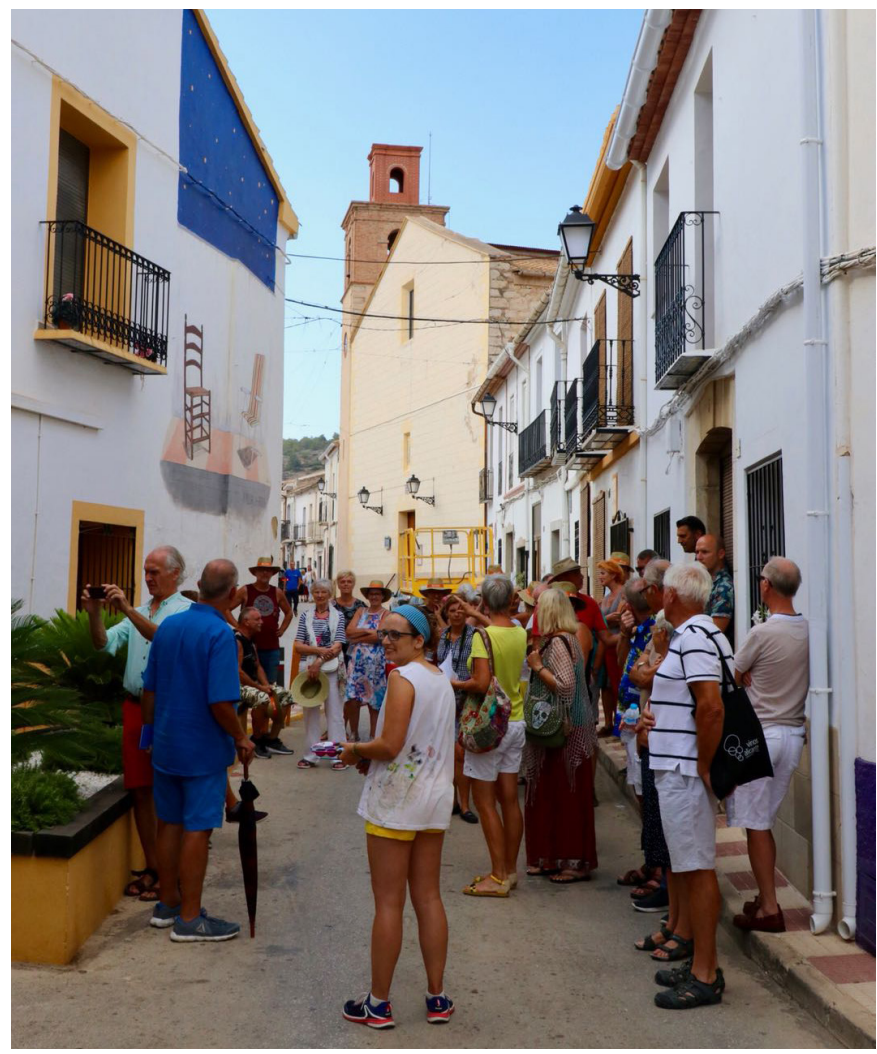

Museo Abierto, proyecto en Senija (Alicante) | foto Tourist Info Vall del Pop 
monio se han quedado pequeños y urge reformularlos para ligarlos a la comunidad, a las emociones, a la educación como aliada y de forma integral. Confirmamos que es necesario buscar formas de gestión relacional, de tejer redes y de extender el conocimiento de las buenas prácticas porque aún queda mucho camino por recorrer, mucho trabajo por hacer para llegar a la gobernanza, muchas mentalidades por abrir para compartir creatividad y memoria, para construir caminos que hagan visibles los patrimonios ocultos, los discursos silenciados y diferentes de los establecido, de lo meramente turístico.

El patrimonio como un proceso en sí nos abre las puertas para reflexionar y trabajar en el marco de un camino siempre inacabado; una reflexión que siempre queda por hacer, porque siempre hay rincones a los que llegar, personas con las que debatir. Esta forma de entender el pasado y la memoria material e inmaterial implica una construcción en continua evolución y transformación; se trata de una construcción viva. Por todo esto seguimos trabajando las buenas prácticas y expandiendo el conocimiento en un camino de ida y vuelta.

\section{NOTAS}

1. Más información del desarrollo del I Encuentro (2018) y la reunión del Pumarejo (2019) podemos encontrarla en revista PH 101 (2020) y revista PH 96 (2019): "Redactívate: mediación y reconocimiento a las personas del patrimonio cultural-local" (https://doi. org/10.33349/2020.101.4729) y "El IAPH bucea y difunde las buenas prácticas para la activación del patrimonio cultural" (https://doi.org/10.33349/2019.96.4356). Asimismo, hay abundante documentación disponible en el Repositorio de Activos Digitales del IAPH (https://repo sitorio.iaph.es/handle/11532/326411; https://repositorio. iaph.es/handle/11532/327676).

\section{Comunidad redactívate}

Grupo evaluador

> Juan José Alarcón Bocanegra, Asociación Los Dólmenes, Valencina (Sevilla).

> Rubén Ballano, Sentir y vivir el patrimonio, Consejería de Educación y Deporte, Junta de Andalucía.

$>$ Rosario Castro, Sentir y vivir el patrimonio, Consejería de Educación y Deporte, Junta de Andalucía.

> Gema Carrera Díaz, IAPH, Consejería de Cultura y Patrimonio Histórico, Junta de Andalucía y Asoc. Andaluza de Antropología.

> Beatriz Castellano Bravo, IAPH, Consejería de Cultura y Patrimonio Histórico, Junta de Andalucía.

> Aniceto Delgado Méndez, IAPH, Consejería de Cultura y Patrimonio Histórico, Junta de Andalucía y Asociación Andaluza de Antropología.

> Víctor Fernández Salinas, Dpto. Geografía Humana, Universidad de Sevilla.

> Yolanda González-Campos, IAPH, Consejería de Cultura y Patrimonio Histórico, Junta de Andalucía.

> Susana Limón Rodríguez, IAPH, Consejería de Cultura y Patrimonio Histórico, Junta de Andalucía.

> Mariela Maitane, Asociación Herstóricas (Granada).

> Nieves Medina Rosales, Proyecto Patrimonio, Aroche (Huelva).

> María Morente del Monte, Museo de Málaga.

> Jaime Moreno Tamarán, Consejería de Cultura y Patrimonio Histórico.

> Ignacio Muñiz Jaén, Ecomuseo del río Caicena, Almedinilla (Córdoba).

> Valle Pérez Cano, IAPH, Consejería de Cultura y Patrimonio Histórico, Junta de Andalucía.

> Myriam Olmedo Morales, IAPH, Consejería de Cultura y

Patrimonio Histórico, Junta de Andalucía.

> Antonio Ortega Ruiz, Universidad Internacional de Andalucía, Baeza (Jaén).

> Maribel Rodríguez Achútegui, Museo de La Rinconada y Asociación para la Interpretación del Patrimonio (AIP).

> Julio Rodríguez Bisquert, IAPH, Consejería de Cultura y Patrimonio Histórico, Junta de Andalucía.

> Carlos Romero Moragas, IAPH, Consejería de Cultura y Patrimonio Histórico, Junta de Andalucía.

> David Ruiz García, Colección Museográfica de Gilena (Sevilla). $>$ Jesús Rull Pereda, Aula de cine, Agencia Andaluza de Instituciones Culturales, Consejería de Cultura y Patrimonio Histórico, Junta de Andalucía.

> Juan Manuel Salas Rojas, Consejería de Agricultura, Pesca y Desarrollo Sostenible, Junta de Andalucía

> Pilar Tassara Andrade, Agencia Andaluza de Instituciones

Culturales, Consejería de Cultura y Patrimonio Histórico, Junta de Andalucía.

> Luz Vega Zabala, Consejería de Agricultura, Pesca y Desarrollo Sostenible, Junta de Andalucía.

> Adrián Yánez, comunicador cultural y Asociación Andaluza de Profesionales de la Gestión Cultural. 